\title{
Entropy coding with Variable Length Re-writing Systems
}

\author{
Herve Jegou \\ University of Rennes \\ Email: Herve.Jegou@irisa.fr
}

\author{
Christine Guillemot \\ IRISA/INRIA \\ Email: Christine.Guillemot@irisa.fr
}

\begin{abstract}
This paper describes a new set of block source codes well suited for data compression. These codes are defined by sets of productions rules of the form $a \bar{l} \rightarrow \bar{b}$, where $a \in \mathcal{A}$ represents a value from the source alphabet $\mathcal{A}$ and $\bar{l}, \bar{b}$ are -small- sequences of bits. These codes naturally encompass other Variable Length Codes (VLCs) such as Huffman codes. It is shown that these codes may have a similar or even a shorter mean description length than Huffman codes for the same encoding and decoding complexity. A first code design method allowing to preserve the lexicographic order in the bit domain is described. The corresponding codes have the same mean description length (mdl) as Huffman codes from which they are constructed. Therefore, they outperform from a compression point of view the $\mathrm{Hu}$ Tucker codes designed to offer the lexicographic property in the bit domain. A second construction method allows to obtain codes such that the marginal bit probability converges to 0.5 as the sequence length increases and this is achieved even if the probability distribution function is not known by the encoder.
\end{abstract}

\section{INTRODUCTION}

Grammars are powerful tools which are widely used in Computer Sciences. Most of lossless compression algorithms can actually be formalized with grammars. Codes explicitly based on grammars have been considered as a mean for data compression [1]. These codes losslessly encode a sequence in two steps. A first analysis step consists in finding the production rules. A second step applies these rules to the sequence to be encoded. These codes have mainly been compared with dictionary-based compression algorithms such as LZ77 [2] or [3], which also implicitly use the grammar formalism. All these codes have in common the fact that the set of production rules depends on the data to be encoded, and not only on the source properties.

In this paper, a new set of codes based on specific production rules is introduced. In contrast with LZ77-like algorithms or grammar codes, the set of production rules is fixed. In contrast with grammar codes introduced so far in the literature, the codes described here encompass Huffman codes [4] (but not Variable-to-Fixed Length codes such as Tunstall Codes [5]). The form of the production rules is presented in Section $\amalg$ The sequence of bits generated by a given production rule may be re-written by a subsequent production rule. They lead to the same encoding and decoding complexity as Huffman codes. A possible drawback of these codes would be that they require backward encoding. However, since most applications deal with block encoding, the forward encoding property is not absolutely required. In Section III] the decoding and encoding procedures with automata will be described. The compression efficiency of these codes will be analyzed in Section IV It is shown in an example that the proposed codes allow for better compression efficiency than Huffman codes.

Two code construction methods are then described. The first method constructs a set of production rules preserving the lexicographic order of the original source sequence in the bit domain. This property is obviously of interest for database applications, since it allows to process comparative queries directly in the bit domain, hence avoiding the prematurate decoding of the compressed dictionary for the query itself. Note that the lexicographic VLC of minimal mdl is usually obtained with the Hu-Tucker algorithm [6]. This algorithm is optimal in the set of VLCs. For some sources, the $\mathrm{Hu}$ Tucker codes may have the same compression efficiency as Huffman codes, but it is not the case in general. The method proposed in Section D constructs lexicographic codes with the same compression performance as Huffman codes and that allow for symbol per symbol encoding and decoding procedures. Obtaining together the properties of lexicographic order preservation and high compression efficiency illustrates the interest of codes based on the proposed set of production rules.

The second construction method described in Section DI] allows to obtain codes, for stationary sources, such that the marginal bit probability is equal to 0.5 . The main advantage of these codes is that this probability is equal to 0.5 even if the actual source probabilities are not known at the encoder, or if the assumed a priori probabilities differ from the true probabilities. Since channel encoders widely assume that $0 s$ and $1 s$ have the same probability, this property is of interest when compressed bitstreams protected by such encoders are transmitted over noisy channels.

\section{Problem statement And Notations}

In the sequel random variables are denoted by upper cases and the corresponding realizations are denoted by lower cases. Sets are denoted by calligraphic characters. The cardinality of a given set $\mathcal{X}$ is denoted $|\mathcal{X}|$. We define $\mathcal{X}^{+}=\bigcup_{i=1}^{\infty} \mathcal{X}^{i}$ and $\mathcal{X}^{*}=\{\varepsilon\} \cup \mathcal{X}^{+}$, where $\varepsilon$ denotes the void sequence. Hence $\mathcal{X}^{*}$ denotes the set of sequences composed of elements of $\mathcal{X}$. Let $\mathbf{S} \in \mathcal{A}^{+}$be a sequence of source symbols taking their values in a finite alphabet $\mathcal{A}=\left\{a_{1}, \ldots a_{i}, \ldots\right\}$. The length of such a sequence is denoted $L(\mathbf{S})$. The alphabet $\mathcal{A}$ is assumed 
to be ordered according to a total order $\prec$. Without loss of generality, we assume that $a_{1} \prec a_{2} \ldots \prec a_{i} \ldots \prec a_{|\mathcal{A}|}$. Let us define $\mathcal{B}=\{0,1\}$. In the sequel, the emitted bitstream is denoted $\mathbf{E}=E_{1} \ldots E_{L(\mathbf{E})} \in \mathcal{B}^{*}$ and its realization is denoted $\mathbf{e}=e_{1} \ldots e_{L(\mathbf{e})}$.

Definition 1: A Variable Length Re-writing System (VLRS) is a set $\mathcal{R}=\bigcup_{i \in \mathcal{A}} \mathcal{R}_{i}$, where $\mathcal{R}_{i}$ denotes the set of rules related to a given symbol $a_{i}$, defined as

$$
\begin{aligned}
& r_{1,1}: \quad a_{1} \bar{l}_{1,1} \quad \rightarrow \quad \bar{b}_{1,1}=b_{1,1}^{1} \ldots b_{1,1}^{L\left(\bar{b}_{1,1}\right)}, \\
& r_{i, j}: \quad a_{i} \bar{l}_{i, j} \quad \rightarrow \quad \bar{b}_{i, j}=b_{i, j}^{1} \ldots b_{i, j}^{L\left(\bar{b}_{i, j}\right)} \\
& r_{|\mathcal{A}|,\left|\mathcal{R}_{|\mathcal{A}|}\right|}: \quad a_{|\mathcal{A}|} \bar{l}_{|\mathcal{A}|,\left|\mathcal{R}_{|\mathcal{A}|}\right|} \quad \rightarrow \quad \bar{b}_{|\mathcal{A}|,\left|\mathcal{R}_{|\mathcal{A}|}\right|}
\end{aligned}
$$

where $\bar{l}_{i, j} \in \mathcal{B}^{*}, \bar{b}_{i, j} \in \mathcal{B}^{+}$. This set is such that

1) $\forall i,\left|\mathcal{R}_{i}\right| \geq 1$,

2) The set $\bigcup_{i=1}^{|\mathcal{A}|} \bigcup_{j=1}^{\left|\mathcal{R}_{i}\right|}\left\{\bar{b}_{i, j}\right\}$ forms a prefix code (i.e. no codeword is the prefix of another [7]).

3) $\forall i, \bigcup_{j=1}^{\left|\mathcal{R}_{i}\right|}\left\{\bar{l}_{i, j}\right\}$ is the set $\{\varepsilon\}$ or forms a full prefix code (i.e, such that the Kraft sum is equal to 1 ).

4) $\forall i \forall i^{\prime} \neq i, \forall j, j^{\prime}, \bar{b}_{i, j}=\bar{l}_{i^{\prime}, j^{\prime}}$ or $\bar{b}_{i, j}$ is not a prefix of $\bar{l}_{i^{\prime}, j^{\prime}}$.

These production rules allow to transform a sequence $\mathbf{s}$ of symbols into a sequence e of bits by successive applications of production rules. These rules are assumed to be reversible: inverting the direction of the arrow allows to recover a given sequence $\mathbf{s}$ from the corresponding bitstream $e$. Note that a given production rule absorbs a symbol $\left(a_{i}\right)$ and some bits $\left(\bar{l}_{i, j}\right)$ from the temporary term to be encoded, and generates a given sequence of bits $\left(\bar{b}_{i, j}\right)$. Huffman codes are covered by this definition. More generally, a VLRS is a Fixed-to-Variable (F-to-V) Length code if $\forall i\left|\mathcal{R}_{i}\right|=1$ and $\bar{l}_{i}=\{\varepsilon\}$.

Example 1: $\operatorname{Code} \mathcal{C}_{1}=\{0,10,11\}$ can be seen as the following VLRS:

$$
\begin{array}{cccc}
r_{1,1}: & a_{1} & \rightarrow & 0 \\
r_{2,1}: & a_{2} & \rightarrow & 10 \\
r_{3,1}: & a_{3} & \rightarrow & 11
\end{array}
$$

Note that Definition 1 does not warranty that such a system leads to a valid prefix code. For example, a rule $r_{i, j}$ where $\bar{b}_{i, j}$ is a prefix of $\bar{l}_{i, j}$ is not valid. In this paper, we focus on VLRS leading to valid codes. Note that Suffix-constrained Codes introduced in [8] form a subset of VLRS and are characterized as follows.

Definition 2: A suffix-constrained code is a VLRS such that $\forall i, j \bar{l}_{i, j}$ is a suffix of $\bar{b}_{i, j}$.

Example 2: The following $\operatorname{VLRS} \mathcal{C}_{2}$ is a suffix-constrained
$C_{1}$
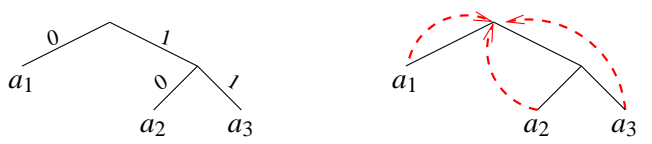

$C_{2}$
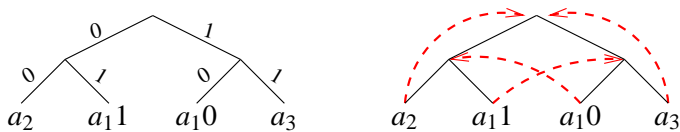

$C_{3}$
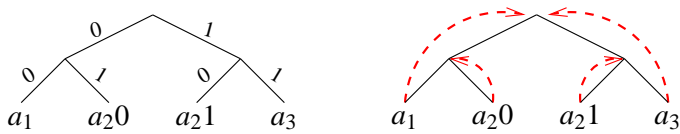

$C_{4}$

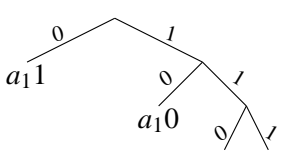

$a_{2} a_{3}$

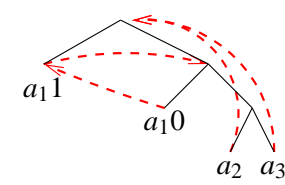

Fig. 1. Examples of VLRS: a VLC $\mathcal{C}_{1}$ and a suffix-constrained code $\mathcal{C}_{2}$. On the right, the transitions triggered by the production rules are depicted by arrows.

code:

$$
\begin{array}{cccc}
r_{1,1}: & a_{1} 0 & \rightarrow & 10 \\
r_{1,2}: & a_{1} 1 & \rightarrow & 01 \\
r_{2,1}: & a_{2} & \rightarrow & 00 \\
r_{3,1}: & a_{3} & \rightarrow & 11
\end{array}
$$

Note that Code $\mathcal{C}_{2}$ can not be encoded in the forward direction. We will come back on this point in Section two following codes will also be considered in the sequel. Note that these codes are not suffix-constrained codes.

Example 3: $\mathcal{C}_{3}$ is defined as

$$
\begin{array}{cccc}
r_{1,1}: & a_{1} & \rightarrow & 00 \\
r_{2,1}: & a_{2} 0 & \rightarrow & 01 \\
r_{2,2}: & a_{2} 1 & \rightarrow & 10 \\
r_{3,1}: & a_{3} & \rightarrow & 11
\end{array}
$$

Example 4: $\mathcal{C}_{4}$ is defined as

$$
\begin{array}{cccc}
r_{1,1}: & a_{1} 1 & \rightarrow & 0 \\
r_{1,2}: & a_{1} 0 & \rightarrow & 10 \\
r_{2,1}: & a_{2} & \rightarrow & 110 \\
r_{3,1}: & a_{3} & \rightarrow & 111
\end{array}
$$

VLRS can also be represented using trees, as depicted in Fig. [1] The tree structure corresponds to the one of the prefix code defined by $\bigcup_{i=1}^{|\mathcal{A}|} \bigcup_{j=1}^{|\mathcal{R}|}\left\{\bar{b}_{i, j}\right\}$. Leaves correspond to both the symbol $a_{i}$ and the sequence of bits $\bar{l}_{i, j}$. 


\section{ENCODING AND DECODING WITH AUTOMATA}

On the encoder side, the purpose of production rules is to transform the sequence $s$ into the sequence e of bits. Any segment of the current sequence (composed of symbols and bits, initialized by s) can be rewritten if there exists a rule having this segment as an input (this input is composed of one symbol and a variable number of bits). When the production rules stop, the sequence contains only bit entities. The set of rules defining a VLRS does not generally allow to encode the sequence $\mathbf{S}$ in the forward direction. Therefore, the encoding must be processed backward. To initiate the encoding process a specific rule must be used to encode the last symbol of the sequence. Indeed the last symbol may not be sufficient to trigger a production rule by itself. In most cases, they can be arbitrarily defined assuming that missing bit(s) equal 0 , at the condition that the termination bit(s) do(es) not trigger a production rule. Hence, the choice 0 is valid for the codes $\mathcal{C}_{1}$, $\mathcal{C}_{2}$ and $\mathcal{C}_{3}$ but should not be used for code $\mathcal{C}_{4}$, since 0 triggers the rule $r_{1,1}$.

Example 5: Let $\mathbf{s}_{\mathbf{1}}=a_{1} a_{2} a_{2} a_{3} a_{2} a_{1} a_{1} a_{1}$ be a sequence of symbols taking their values in the alphabet $\mathcal{A}_{1}=\left\{a_{1}, a_{2}, a_{3}\right\}$. This sequence is encoded with Code $\mathcal{C}_{2}$. Since the last symbol is $a_{1}$, no rule applies directly. Therefore, the termination bit 0 is concatenated to this sequence in order to initiate the encoding. The encoding then proceeds as follows:

$\begin{array}{rr}r_{1,1}: & \mathbf{s}_{\mathbf{1}} 0=a_{1} a_{2} a_{2} a_{3} a_{2} a_{1} a_{1} a_{1} 0 \\ r_{1,2}: & a_{1} a_{2} a_{2} a_{3} a_{2} a_{1} \underline{a_{1} 10} \\ r_{1,1}: & a_{1} a_{2} a_{2} a_{3} a_{2} \underline{a_{1} 01} 0 \\ r_{2,1}: & a_{1} a_{2} a_{2} a_{3} \underline{a_{2} 1010} \\ r_{3,1}: & a_{1} a_{2} a_{2} a_{3} 001010 \\ r_{2,1}: & a_{1} a_{2} a_{2} 11001010 \\ r_{2,1}: & a_{1} a_{2} 0011001010 \\ r_{1,1}: & a_{1} 000011001010 \\ & \mathbf{e}_{\mathbf{1}}=1000011001010\end{array}$

In [8], it was shown that transmitting the termination bit is not required for suffix-constrained codes, as shown in Example [5] This is due to the fact that a bit generated by a production rule of a suffix-constrained code will not be modified by a subsequent production rule. Since these termination bits may be required in the general case, it will be assumed that they are known at the decoder. In the following example, the termination bit must be 1 . Note that the sequence is encoded with less than 1 bit per symbol.

Example 6: Let us now consider the sequence $\mathbf{s}_{\mathbf{1}}^{\prime}=$ $a_{1} a_{1} a_{1} a_{1} a_{1}$. This sequence is encoded with code $\mathcal{C}_{4}$ as

$$
\begin{array}{rr}
r_{1,1}: & \mathbf{s}_{\mathbf{1}}^{\prime} 1=a_{1} a_{1} a_{1} a_{1} \underline{a_{1} 1} \\
r_{1,2}: & a_{1} a_{1} a_{1} \underline{a_{1} 0} \\
r_{1,1}: & a_{1} a_{1} \underline{a_{1} 10} \\
r_{1,2}: & a_{1} \underline{a_{1} 0} 0 \\
r_{1,1}: & \underline{a_{1} 10} 0 \\
& \mathbf{e}_{\mathbf{1}}^{\prime}=000
\end{array}
$$

On the decoder side, the decoding is processed forward
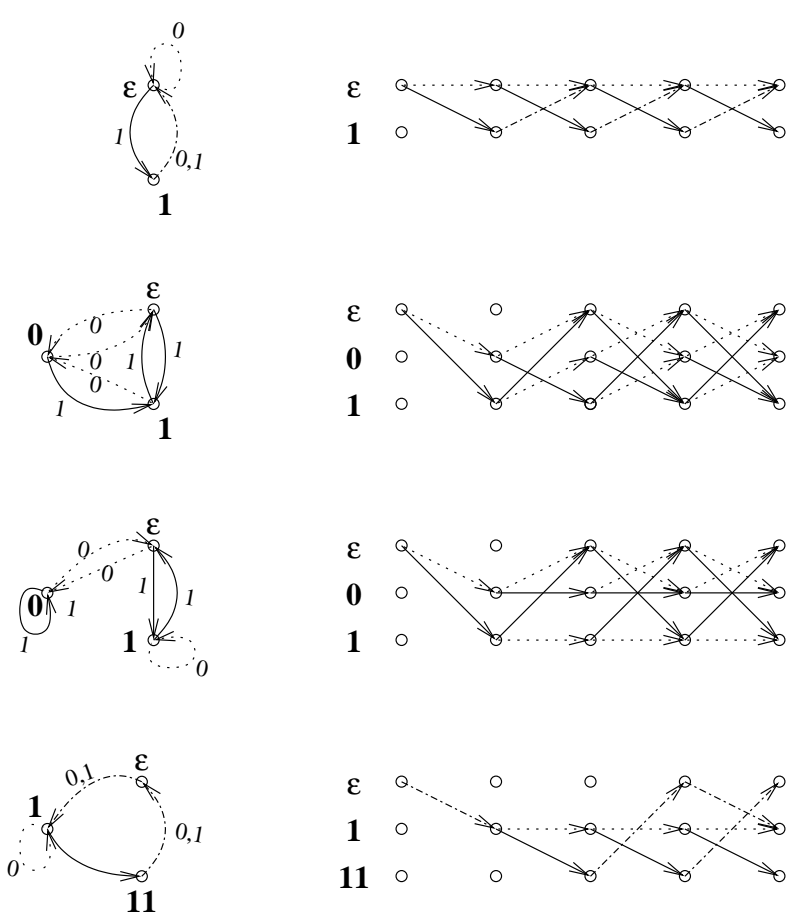

Fig. 2. Decoding automata corresponding to the codes $\mathcal{C}_{1}, \mathcal{C}_{2}, \mathcal{C}_{3}$ and $\mathcal{C}_{4}$ and corresponding decoding trellises. Transitions corresponding to $0 s$ and $1 s$ are respectively plotted with dotted and solid lines.

using reverse rules. The encoding and decoding algorithms are implemented using automata. These automata are used to catch the memory of the encoding and decoding processes. This memory corresponds to a segment of bits that may be useful for the next production rule. Hence, they are obtained directly from the set of production rules. The transitions on the automaton representing the encoding process are triggered by symbols. The internal states of the automaton are given by the variable length segments of bits $\left\{\bar{l}_{i, j}\right\}$. This automaton may be reduced if a variable length bit segment $\bar{l}_{i, j}$ is a prefix of another segment $\bar{l}_{i^{\prime}, j^{\prime}}$ (in that case, according to Definition 1 we have $i \neq i^{\prime}$ ). If $\forall i, j, \bar{l}_{i, j}=\varepsilon$, there is only one internal state $\{\varepsilon\}$ for the encoding automaton corresponding to code $\mathcal{C}_{1}$. The sets of states of encoding automata of codes $\mathcal{C}_{2}, \mathcal{C}_{3}$ and $\mathcal{C}_{4}$ are identical and are equal to $\{0,1\}$.

The states of the decoding automata correspond to bit segments that have already been decoded, but which are not sufficient to identify a symbol. For VLCs such as Huffman codes, these internal states correspond to the internal nodes of the decoding codetree.

Example 7: The set of internal states of codes $\mathcal{C}_{1}, \mathcal{C}_{2}, \mathcal{C}_{3}$ and $\mathcal{C}_{4}$ are respectively $\{\varepsilon, 1\},\{\varepsilon, 0,1\},\{\varepsilon, 0,1\}$ and $\{\varepsilon, 1,11\}$.

The graphical representations of the decoding automata may be deduced from the tree representations given in Fig. 1 These automata are depicted in Fig. 2] The decoding trellises corresponding to these automata are depicted on the right. For sake of clarity, the symbols generated by the bit transitions are not shown. However, note that the set of generated symbol(s) 
must also be associated to each bit transition. For the codes $\mathcal{C}_{1}$, $\mathcal{C}_{2}$ and $\mathcal{C}_{3}$, at most 1 symbol is associated to each bit transition. It is not the case for Code $\mathcal{C}_{4}$, where the transition starting from decoding state 1 triggered by the bit 0 generates the symbol $a_{1}$ twice. As shown in Example 6 and demonstrated in Section IV this transition allows to encode long sequences of $a_{1}$ with less than 1 bit, at the cost of a higher encoding cost for the symbols $a_{2}$ and $a_{3}$.

\section{COMPRESSION EFFICIENCY}

In this section, we analyse the compression efficiency of VLRSs. Let us assume that $\mathbf{S}$ is a memoryless source characterized by its stationary probability distribution function (pdf) on $\mathcal{A}: \boldsymbol{\mu}=\left\{\mathbb{P}\left(a_{1}\right), \ldots \mathbb{P}\left(a_{i}\right), \ldots\right\}$. Let

$$
\delta\left(r_{i, j}\right)=L\left(\bar{b}_{i, j}\right)-L\left(\bar{l}_{i, j}\right)
$$

denote the number of bits generated by a given production rule $r_{i, j}$. Note that for the particular case where $\forall i, \forall j, j^{\prime} \delta\left(r_{i, j}\right)=$ $\delta\left(r_{i, j^{\prime}}\right)$, the mdl is equal to $\sum_{a_{i} \in \mathcal{A}} \mathbb{P}\left(a_{i}\right) \delta_{i, 1}$.

Example 8: Let us assume that $\mathbf{S}$ is a memoryless source of pdf $\boldsymbol{\mu}_{1}=\{0.7,0.2,0.1\}$. The entropy of this source is 1.157 . The mdl of Code $\mathcal{C}_{1}$ is equal to 1.3. For the code $\mathcal{C}_{2}$, we have $\delta\left(r_{1,1}\right)=\delta\left(r_{1,2}\right)=1$ and $\delta\left(r_{2,1}\right)=\delta\left(r_{3,1}\right)=2$. The mdl of this code is also equal to 1.3 .

Let $R_{t}: S_{t} \bar{L}_{t} \rightarrow \bar{B}_{t}$ denote the rule to be used in order to encode a given symbol $S_{t}$. Since the encoder proceeds backward and since the source $\mathbf{S}$ is memoryless, the process $\left(Z_{t^{\prime}}\right)=\left(R_{L(\mathbf{S})}, \ldots R_{L(\mathbf{S})}, \ldots R_{1}\right)$ obtained from the process $\left(R_{t}\right)$ by reversing the symbol clock $t$, i.e. $\left(Z_{t^{\prime}}\right)_{t^{\prime}=1, \ldots L(\mathbf{S})}=$ $\left(R_{L(\mathbf{S})-t+1}\right)_{t=1, \ldots L(\mathbf{S})}$, forms an invariant Markov chain. In other words we have $\mathbb{P}\left(Z_{t^{\prime}} \mid Z_{1}, \ldots Z_{t^{\prime}-1}\right)=\mathbb{P}\left(Z_{t^{\prime}} \mid Z_{t^{\prime}-1}\right)=$ $\mathbb{P}\left(R_{t} \mid R_{t+1}, \ldots R_{L(\mathbf{S})}\right)=\mathbb{P}\left(R_{t} \mid R_{t+1}\right)=\mathbb{P}\left(R_{L(\mathbf{S})-1} \mid R_{L(\mathbf{S})}\right)$. If $S_{t}=a_{i}$, the rule $r_{i, j}$ is triggered if and only if the realization of $\bar{L}_{t}$ is a prefix of the bits $\bar{B}_{t+1}$ generated by the previous production rule. As a consequence, the probability $\mathbb{P}\left(R_{t} \mid R_{t+1}\right)$ can be deduced from the source pdf as

$$
\begin{aligned}
\mathbb{P}\left(R_{t}=r_{i, j} \mid\right. & \left.R_{t+1}=r_{i^{\prime}, j^{\prime}}\right) \\
& =\mathbb{P}\left(R_{t}=r_{i, j} \mid \bar{B}_{t+1}=\bar{b}_{i^{\prime}, j^{\prime}}\right) \\
& =\mathbb{P}\left(S_{t}=a_{i}, \bar{L}_{t}=\bar{l}_{i, j} \mid \bar{B}_{t+1}=\bar{b}_{i^{\prime}, j^{\prime}}\right) \\
& = \begin{cases}\mathbb{P}\left(a_{i}\right) & \text { if } \bar{l}_{i, j} \text { is prefix of } \bar{b}_{i^{\prime}, j^{\prime}}, \\
0 & \text { otherwise. }\end{cases}
\end{aligned}
$$

Assuming that $\left(Z_{t^{\prime}}\right)$ is irreducible and aperiodic, the marginal probability distribution $\mathbb{P}\left(Z_{t^{\prime}}=r_{i, j}\right)$ is obtained from the transition matrix $\mathbb{P}\left(Z_{t^{\prime}} \mid Z_{t^{\prime}-1}\right)$ as the normalized eigenvector associated to the eigenvalue 1. As $t^{\prime}$ grows to infinity (which requires that $t \rightarrow \infty$ ), the expectation of $\delta\left(Z_{t^{\prime}}\right)$ is the expectation of the number of bits generated by a production rule. With the Cesaro theorem, it also provides the asymptotic value of the mdl as the sequence length increases.

Example 9: For the code $\mathcal{C}_{4}$, the transition matrix correspond- ing to the source pdf of Example 8 is

$\left[\begin{array}{cccc}0 & 0.7 & 0.7 & 0.7 \\ 0.7 & 0 & 0 & 0 \\ 0.2 & 0.2 & 0.2 & 0.2 \\ 0.1 & 0.1 & 0.1 & 0.1\end{array}\right]$

which leads to $\mathbb{P}\left(R_{t}=r_{i, j}\right)=\{0.412,0.288,0.2,0.1\}$. Finally, the mdl of this code is $\operatorname{mdl}\left(\mathcal{C}_{4}\right)=0.412 \times 0+0.288 \times$ $1+0.2 \times 3+0.1 \times 3=1.188$.

The mdl obtained in Example 9 is much closer to the entropy than the mdl obtained with Huffman codes. The expected number of bits required to code the symbol $a_{1}$ is less than 0.5 bit. One can also process the exact mdl of a VLRS for sequences of finite length. Indeed, the expectation of the number of termination bit(s) as well as the pdf $\mathbb{P}\left(R_{t}=\right.$ $\left.r_{i, j} \mid t=L(\mathbf{S})\right)$ of the last rule can be obtained from the termination bit choice and from the source pdf. The exact probability $\mathbb{P}\left(R_{t}=r_{i, j} \mid t=\tau\right)$ of having a given rule for a given symbol clock $\tau$ can then be computed and subsequently one can deduce the expectation of the number of bits generated to encode the symbol $S_{\tau}$.

\section{LeXICOGRAPHIC CODE DESIGN}

This section describes a VLRS construction method which allows to preserve the lexicographic order of the source alphabet in the bit domain. As a starting point, we assume that the Huffman code corresponding to the source pdf $\boldsymbol{\mu}$ is already known. The length of the Huffman codeword associated to the symbol $a_{i}$ is denoted $k_{i}$. Let $k^{+}=\max _{i} k_{i}$ denote the length of the longest codeword. First, let us underline that the union $\bigcup_{i, j}\left\{\bar{b}_{i, j}\right\}$ of all the bit sequences $\bar{b}_{i, j}$ will form a Fixed Length Code (FLC) $\mathcal{F}$ of length $k^{+} \cdot \mathcal{F}$ contains $2^{k^{+}}$ codewords. These codewords will be assigned to productions rules in the lexicographic order. Starting with the smaller symbol $a_{1}, 2^{k^{+}-k_{i}}$ rules are defined for symbol $a_{i}$. The left part of these rules are defined so that the set $\left\{\bar{l}_{i, j}\right\}_{j \in\left[1 . .\left|\mathcal{R}_{i}\right|\right]}$ forms a FLC of length $k^{+}-k_{i}$. If $k_{i}=k^{+}$, this FLC only contains the element $\varepsilon$. The $2^{k^{+}-k_{i}}$ smallest remaining codewords of $\mathcal{F}$, i.e. those which have not been assigned to previous symbols of $\mathcal{F}$, are then assigned to these productions rules so that $\forall j, \bar{l}_{i, j} \leq \bar{l}_{i, j^{\prime}} \Rightarrow \bar{b}_{i, j} \leq \bar{b}_{i, j^{\prime}}$. By construction, the proposed algorithm leads to a VLRS with the lexicographic property and with the same compression efficiency as the code from which it is constructed. In some cases, the set of production rules generated in previous steps may be simplified.

Example 10: Let us now assume that the source $\mathbf{S}$ is memoryless of pdf $\boldsymbol{\mu}_{2}=\{0.2,0.7,0.1\}$. Since $a_{2}$ has the highest probability, the Huffman code $\mathcal{H}_{2}=\{10,0,11\}$ corresponding to this pdf is not lexicographic. The $\mathrm{Hu}$-Tucker code associated to this source is the code $\mathcal{C}_{1}$ proposed in Example 1 and its mdl is equal to 1.8 .

The VLRS is constructed according to the proposed construction procedure. For $\mathcal{H}_{2}$, we have $k_{2}=1$ and $k_{1}=k_{3}=$ $k^{+}=2$. Hence $\mathcal{F}=\{00,01,10,11\}$. Since $k_{1}=2$, only 1 production rule $r_{1,1}$ is assigned to the symbol $a_{1}$ and $b_{1,1}=\varepsilon$, 
$C_{1}$
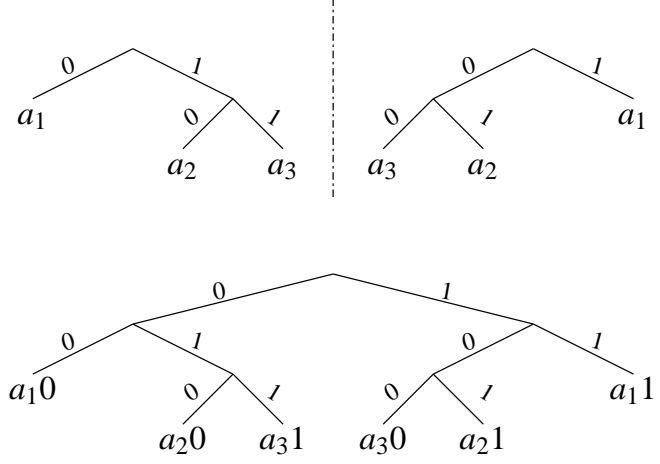

Fig. 3. Primitive code $\mathcal{C}_{1}$, its opposite $\tilde{\mathcal{C}}_{1}$ and the resulting mirror VLRS.

which implies $r_{1,1}: a_{1} \rightarrow 00$. The symbol $a_{1}$ is then assigned two production rules $r_{2,1}$ and $r_{2,2}$ so that $r_{2,1}: a_{2} 0 \rightarrow 01$ and $r_{2,2}: a_{2} 1 \rightarrow 10$. The construction algorithm finishes with the assignment of rule $r_{3,1}: a_{3} \rightarrow 11$ to symbol $a_{3}$. Finally, we obtain the code $\mathcal{C}_{3}$ proposed in Example 3 for which the $\mathrm{mdl}$ is equal to 1.3 together with the lexicographic property.

Although the proposed construction allows to obtain lexicographic codes with the same compression efficiency as Huffman codes, it does not construct, in general, the best lexicographic VLRS from a compression efficiency point of view. One may find some lexicographic VLRS with lower mdl.

\section{Mirror Code Design}

The code design described in this section allows to obtain codes with bit marginal probabilities that are asymptotically equal to 0.5 as the sequence length increases. Let us again assume, as a starting point, that we know a VLC code $\mathcal{H}=\left\{\bar{b}_{1,1}, \ldots \bar{b}_{|\mathcal{A}|, 1}\right\}$. Let us now consider the code $\tilde{\mathcal{H}}=$ $\left\{\tilde{\bar{b}}_{1,1}, \ldots \tilde{\bar{b}}_{|\mathcal{A}|, 1}\right\}$ defined so that each bit transition of the codetree characterizing $\tilde{\mathcal{H}}$ is the opposite value from the corresponding bit transition in $\mathcal{H}$, as depicted in Fig. 3

The VLRS is obtained by putting together these two codes. The codes $\mathcal{H}$ and $\tilde{\mathcal{H}}$ are respectively used to define the two sets of $|\mathcal{A}|$ production rules forming the new VLRS as

$$
\mathcal{M}=\left\{\begin{array}{l}
\left\{a_{i} b_{i, 1}^{L\left(\bar{b}_{i, 1}\right)} \rightarrow 0 \bar{b}_{i, 1}\right\}_{i \in[1 . .|\mathcal{A}|]} \\
\left\{a_{i} \tilde{b}_{i, 1}^{L\left(\tilde{\bar{b}}_{i, 1}\right)} \rightarrow 1 \tilde{\bar{b}}_{i, 1}\right\}_{i \in[1 . .|\mathcal{A}|]} .
\end{array}\right.
$$

Note that the production rules associated to codes $\mathcal{H}$ and $\tilde{\mathcal{H}}$ respectively define the subtrees corresponding to bit transitions 0 and 1 . Note also that the resulting code, by construction, is a suffix-constrained code.

Example 11: The construction associated to the code $\mathcal{C}_{1}$ leads to the following VLRS:

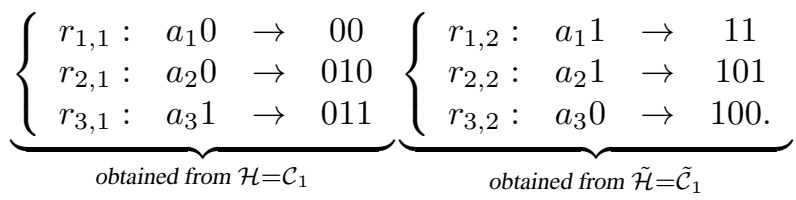

Proof of $\forall n, \lim _{L(\mathbf{S}) \rightarrow \infty} \mathbb{P}\left(E_{n}=0\right)=0.5$ : Let us consider a VLRS $\mathcal{M}$ constructed according to the previous guidelines. The notation $b_{i, j}$ refers to this VLRS (not to the VLC from which it is constructed). Let $f_{t}=\mathbb{P}\left(B_{t}^{1}=0\right)$ denote the marginal bit probability associated to the first bit generated by a given production rule. Since the VLRS is constructed from a VLC, we have $\forall i, j, \delta\left(r_{i, j}\right) \geq 1$, which means that every rule produces at least one bit. The value $f_{t}$ can be written as

$$
\begin{aligned}
f_{t} & =\sum_{i \in[1 . .|\mathcal{A}|], j \in[1 . .2]} \mathbb{P}\left(R_{t}=r_{i, j}, B_{t}^{1}=0\right) \\
& =\sum_{i \in[1 . .|\mathcal{A}|], j=1} \mathbb{P}\left(S_{t}=a_{i}, B_{t+1}^{1}=l_{i, 1}^{L\left(l_{i, 1}\right)}\right) \\
& =\sum_{i \in[1 . .|\mathcal{A}|], j=1} \mathbb{P}\left(S_{t}=a_{i}, L_{t}^{L\left(L_{t}\right)}=0\right) f_{t+1} \\
& +\sum_{i \in[1 . .|\mathcal{A}|], j=1} \mathbb{P}\left(S_{t}=a_{i}, L_{t}^{L\left(L_{t}\right)}=1\right)\left(1-f_{t+1}\right) .
\end{aligned}
$$

Let $\alpha=\sum_{a_{i} \in \mathcal{A}} \mathbb{P}\left(a_{i}, l_{i, 1}^{L\left(\bar{l}_{i, 1}\right)}=0\right)$. This entity corresponds to the sum of the probabilities of the symbols to which a codeword ending with 0 has been assigned. Note that $0<$ $\alpha<1$. Inserting this entity in Eqn. 6 we obtain

$$
f_{t}=\alpha f_{t+1}+(1-\alpha)\left(1-f_{t+1}\right) .
$$

We can now study the asymptotic behavior of this sequence as $t^{\prime}=L(\mathbf{S})-t+1$ tends to $+\infty$ (note that $f_{L(\mathbf{S})}$ is a constant). The absolute value of the derivative of the function $g(x)=\alpha x+(1-\alpha)(1-x)$ is strictly lower than 1 when $0<$ $\alpha<1$. Consequently, the fixed-point theorem applies and the sequence $f_{L(\mathbf{S})}, f_{L(\mathbf{S})-1}, \ldots f_{t^{\prime}}$ converges to the solution of $x=g(x)$, which is 0.5 . Subsequently, $\forall i$, opposite codewords $\bar{b}_{i, 1}$ and $\bar{b}_{i, 2}$ are equiprobable, which concludes the proof.

\section{Conclusion and Perspectives}

VLRSs have a low encoding and decoding complexity, allowing for instantaneous decoding and may have a lower mdl than Huffman codes. The degree of freedom that they offer allows to design codes with interesting properties, as shown in sections $\nabla$ and VI Hopefully, the design of $\nabla \mathbf{D}$ may lead to soft decoding results outperforming the ones obtained with source codes with a marginal bit probability not equal to 0.5 .

\section{REFERENCES}

[1] H. Kieffer and E.-H. Yang, "Grammar-based codes: a new class of universal lossless source codes," IEEE Trans. Inform. Theory, vol. 46, pp. 737-754, 2000.

[2] J. Ziv and A. Lempel, "A universal algorithm for data compression," IEEE Trans. Inform. Theory, vol. 23, pp. 337-343, 1977.

[3] - "Compression of individual sequences via variable-rate coding," IEEE Trans. Inform. Theory, vol. 24, pp. 530-343, 1978.

[4] D. Huffman, "A method for the construction of minimum redundancy codes," in Proc. of the IRE, vol. 40, 1952, pp. 1098-1101.

[5] B. Tunstall, "Synthesis of noiseless compression codes," Ph.D Dissertation, Georgia Institute of Technology, Atlanta, 1967.

[6] T. C. Hu and A. C. Tucker, "Optimal computer search trees and variable length alphabetic codes,” SIAM J. Appl. Math., pp. 514-532, 1971.

[7] T. M. Cover and J. A. Thomas, Elements of Information Theory. John Wiley, 1991, ch. 5, pp. 78-124.

[8] H. Jégou and C. Guillemot, "Suffix-constrained codes for progressive and robust data compression," in Proc. EUSIPCO, Sept. 2004, vienna. 